\title{
Electrical conductivity measurements on gel grown KDP crystals added with urea and thiourea
}

\author{
M PRIYA ${ }^{\dagger}$, C M PADMA ${ }^{\dagger}$, T H FREEDA, C MAHADEVAN ${ }^{*}$ and C BALASINGH ${ }^{\dagger}$ \\ Department of Physics, S.T. Hindu College, Nagercoil 629 002, India \\ ${ }^{\dagger}$ Scott Christian College, Nagercoil 629 003, India
}

MS received 4 September 2000; revised 16 July 2001

\begin{abstract}
Pure and impurity added (with urea and thiourea) KDP single crystals were grown by the gel method using silica gels. Electrical conductivity measurements were carried out along both the unique axis and perpendicular directions at various temperatures ranging from 30 to $140^{\circ} \mathrm{C}$ by the conventional two-probe method. The present study shows that the conductivity in KDP crystals, for both the impurities considered, increases with the increase in impurity concentration and temperature. Activation energies were also determined and reported.
\end{abstract}

Keywords. Impurity added KDP crystals; gel method; electrical conductivity; activation energy.

\section{Introduction}

Potassium dihydrogen orthophosphate (KDP), $\mathrm{KH}_{2} \mathrm{PO}_{4}$, is an interesting material and, due to that fact, several research workers have shown considerable interest on it (Varma et al 1983; Henisch 1988; Rashkovich 1991; Udupa et al 1997; Freeda and Mahadevan 2000). A research programme on the growth and physical properties of pure and impurity added KDP crystals is being carried out in this laboratory. As a part of the programme, we have studied the effect of organic impurities [urea, a nonlinear optical material, and thiourea, a ferroelectric material, added heavily (impurity added in the KDP solution used for the growth of crystals) with impurity concentration ranging from 2000 to $10000 \mathrm{ppm}$, i.e. $0 \cdot 2$ to 1.0 mole $\%$ ] on the d.c. electrical conductivity at various temperatures ranging from 30 to $140^{\circ} \mathrm{C}$ of KDP crystals grown by the gel method. We report here the results obtained in our present study.

\section{Experimental}

Single crystals were grown by using the procedures reported earlier (Freeda and Mahadevan 2000). Analytical reagent (AR) grade samples of the required chemicals (KDP, urea and thiourea) along with double distilled water and ethyl alcohol were used. KDP was added with urea and thiourea separately each in six different KDP: impurity molecular ratios viz. $1: 0.000$ (pure KDP), $1: 0 \cdot 002$, $1: 0 \cdot 004,1: 0 \cdot 006,1: 0 \cdot 008,1: 0 \cdot 010$.

\footnotetext{
*Author for correspondence
}

Scalenohedral (twelve sided polyhedron) morphology was exhibited by all the crystals grown. All the crystals grown were found to be very stable, colourless and transparent.

Crystals with high transparency and large defect-free size $(>3 \mathrm{~mm})$ were selected and used for the electrical conductivity measurements. The extended portions of the crystals were removed completely and the opposite faces were polished and coated with good quality graphite to obtain a good ohmic contact.

The d.c. conductivity measurements were carried out along both the unique axis $(c-)$ and perpendicular ( $a$ - and $b$-) directions for all the eleven crystals grown using the conventional two-probe technique at various temperatures ranging from 30 to $140^{\circ} \mathrm{C}$. The dimensions of the crystals were measured using a travelling microscope (L.C. = $0.001 \mathrm{~cm})$. The conductivity $(\sigma)$ of the crystal was calculated using the relation

$$
\sigma=\frac{d}{R A}
$$

where $R$ is the measured resistance, $d$ the thickness of the sample crystal and $A$ the area of the face of the crystal in contact with the electrode. Inaccuracy involved in this measurement was within $\pm 4 \%$.

Plots between $\ln (\sigma)$ and $1000 / T$ were found to be very nearly linear. So the conductivity values can be fitted to the relation

$$
\sigma=\sigma_{0} \exp (-E / k T)
$$

where $E$ is the activation energy, $k$ the Boltzmann constant, $T$ the absolute temperature and $\sigma_{0}$ the parameter depending on the material. Activation energies were 
estimated using the slopes of the above line plots $(E=-$ slope $\times k)$.

When the crystals are grown in gel media, there is a possibility that the conductivity and related properties are different for crystals even though they are grown in the same container. It would be better if there is a possibility to estimate quantitatively the concentration level of the impurity that is present in each crystal. But, it is very difficult to do so since the impurities considered in the present study are organic compounds without any metal atom. Hence, in order to understand qualitatively whether the added impurity has entered into the KDP lattice or not, we carried out the density measurement by using the flotation technique.

As it was difficult to determine the impurity concentration in the crystal, no experiment was carried out to determine this. However, we followed an approximate method available in the literature (Krishnaswamy 1984) to do the same.

If $X$ and $Y$ are the initial concentration $(\mathrm{g} / 100 \mathrm{cc})$ of $A$ and $B$ the final stoichiometry will be $\left(X-A_{x}\right):\left(Y-A_{y}\right)$, where $A_{x}$ and $A_{y}$ are the solubilities of components $A$ and

Table 1. Densities and activation energies.

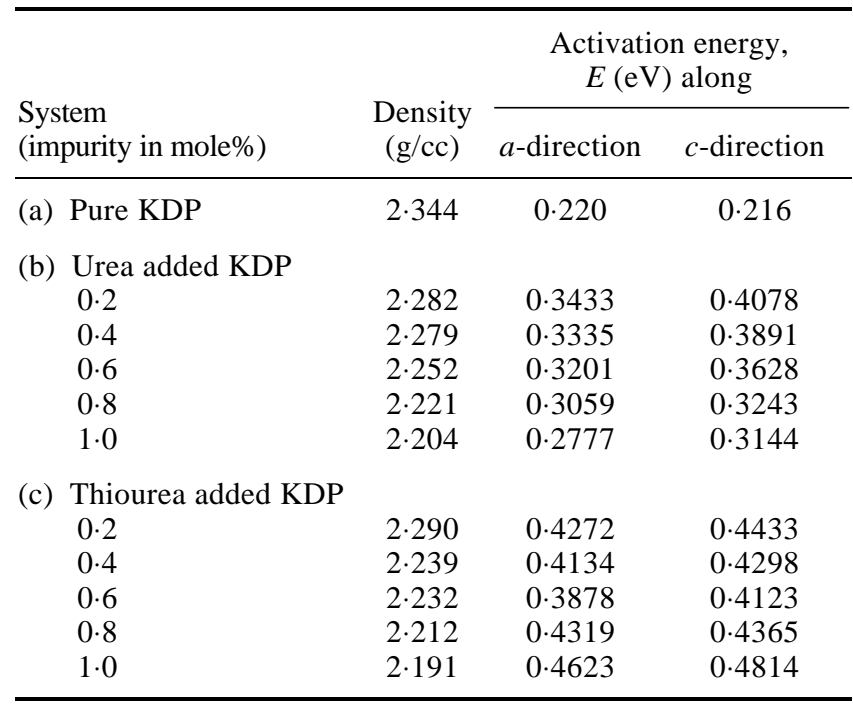

Table 2. Estimated impurity concentrations.

\begin{tabular}{lcc}
\hline & \multicolumn{2}{c}{$\begin{array}{c}\text { Estimated impurity concentration } \\
\text { in the crystal (mole\%) }\end{array}$} \\
\cline { 2 - 3 } $\begin{array}{l}\text { Impurity concentration } \\
\text { in the solution (mole\%) }\end{array}$ & $\begin{array}{c}\text { For urea } \\
\text { added KDP }\end{array}$ & $\begin{array}{c}\text { For thiourea } \\
\text { added KDP }\end{array}$ \\
\hline 0.2 & 0.056 & 0.076 \\
0.4 & 0.144 & 0.189 \\
0.6 & 0.235 & 0.301 \\
0.8 & 0.321 & 0.413 \\
1.0 & 0.409 & 0.524 \\
\hline
\end{tabular}

$B$, respectively. If we simply use this relation, in the present study, we would get negative value for $\left(Y-A_{y}\right)$. So, in order to avoid this situation, we modified the above ratio as

$$
\left(10000 X-A_{x}\right):\left(10000 Y-A_{y}\right)
$$

The values obtained are expected to be reasonable ones and any inaccuracy if present will be negligible.

\section{Results and discussion}

It was observed that the difference in densities of crystals grown in the same container was very small and negligible. Average densities are given in table 1 . The value observed for pure KDP $(2.344 \mathrm{~g} / \mathrm{cc})$ compares well with that reported in the literature $(2 \cdot 338 \mathrm{~g} / \mathrm{cc})$ (Dean 1979). For both the impurities considered in the present study, the observed decrease of density of KDP crystal caused by the impurities indicates that the impurities have entered into the lattice of KDP crystals. Moreover, it can be seen that the density decreases further with the increase in impurity concentration of the aqueous solution of KDP used for the growth of crystals.

The estimated impurity concentration values are presented in table 2 . In real crystals, concentration of

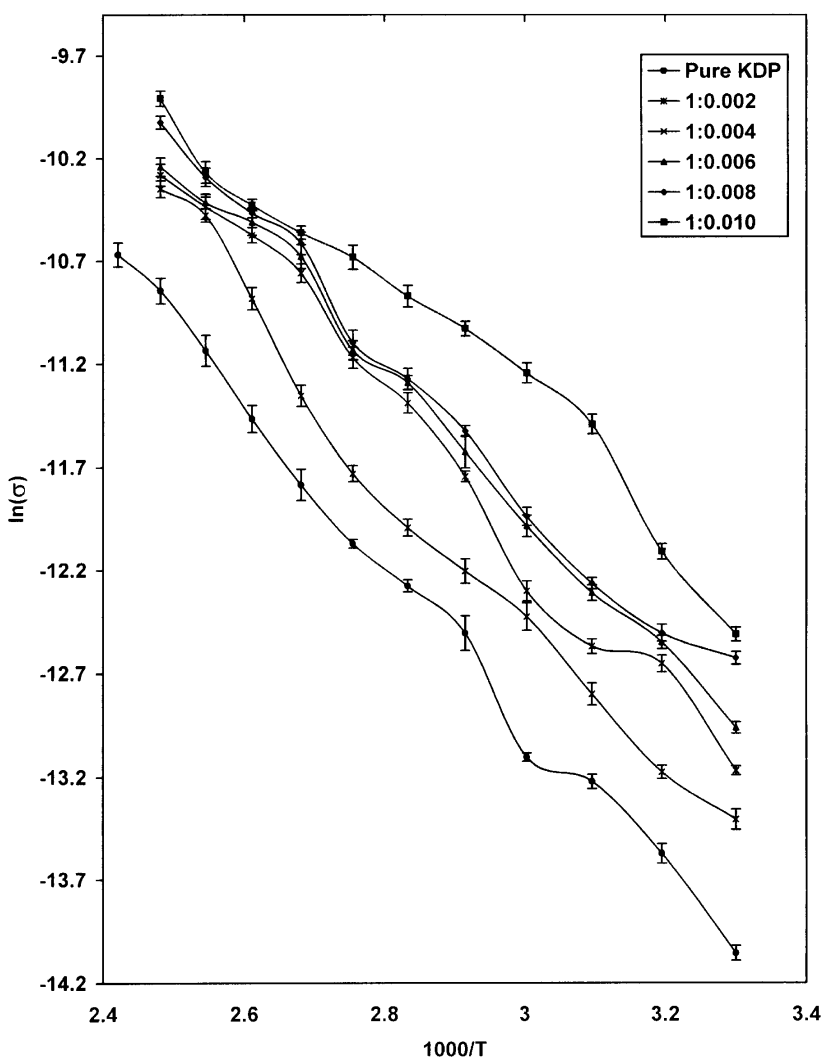

Figure 1. Variation of $\ln (\sigma)$ with $1000 / T$ for urea added KDP along $a$-direction. 
interstitials is expected to be of the order of $10^{15}-10^{20} \mathrm{~cm}^{-3}$, i.e. up to a maximum of $1 \%$ (Here concentration of molecules is assumed as $10^{22} \mathrm{~cm}^{-3}$ ). In the present study, the estimated impurity concentrations show that the impurity in the crystal is mainly occupying the interstitial positions.

The $\sigma$ values obtained along the two directions ( $a$ - and $b$-) perpendicular to the unique axis (c-direction) are, within experimental error, the same. Figures $1-4$ show the plots of $\ln (\sigma)$ against $1000 / T$ for the pure and impurity added KDP crystals. The values of the activation energy, $E$ for pure and impurity added KDP crystals are given in table 1 .

Conductivities obtained in the present study are of the same order with those obtained by previous workers for the KDP crystals $\left(\times 10^{6}\right.$ mho/meter) (Harris and Vella 1966; Shanmugham et al 1985; Ramasubramanian and Mahadevan 1991; Udupa et al 1997; Shanthini et al 1999; Freeda and Mahadevan 2000).

It can be seen that, for both the impurities considered in the present study, the electrical conductivity increases with the increase in impurity concentration and temperature. This is similar to that observed for oxalate (Shanmugham et al 1985) and ammonium compound (Freeda and Mahadevan 2000) added KDP crystals. The defect concentration will increase exponentially with tempera-

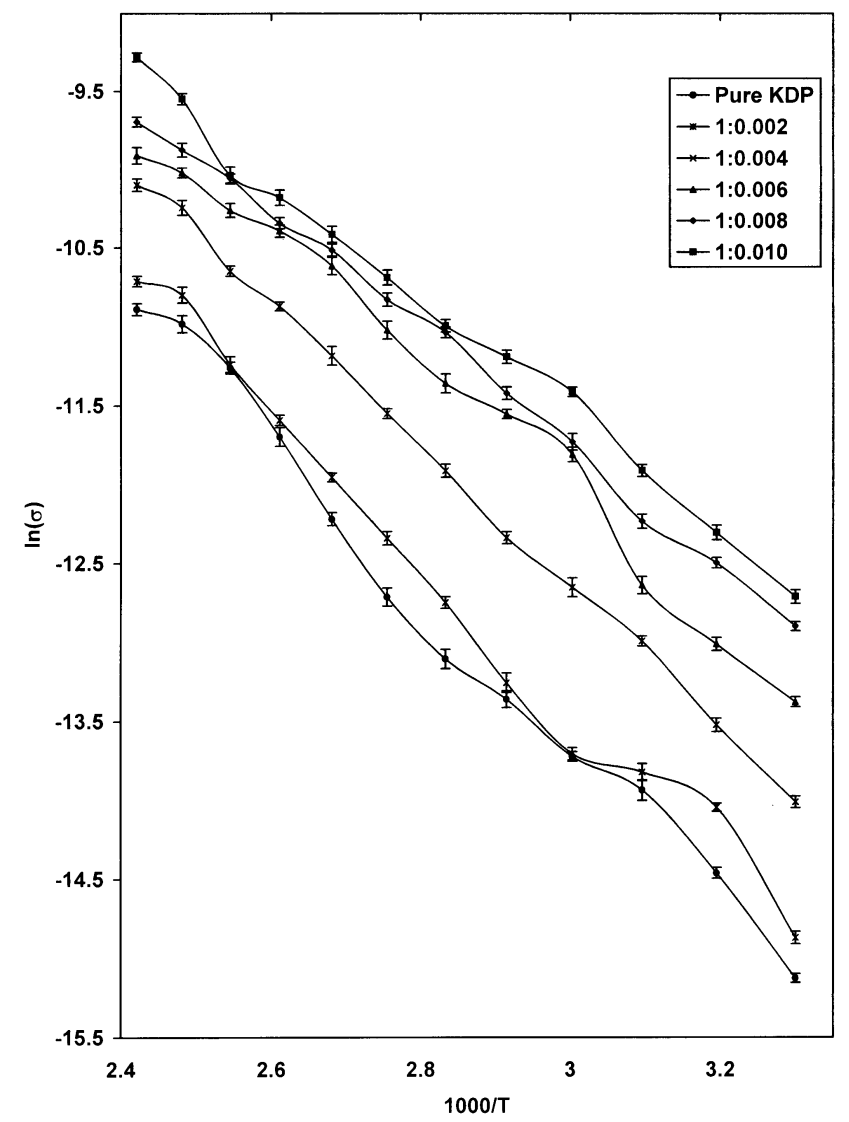

Figure 2. Variation of $\ln (\sigma)$ with $1000 / T$ for urea added KDP along $c$-direction. ture and consequently the electrical conduction also increases. The addition of impurity further increases the electrical conduction in the temperature region considered.

As the impurities mainly occupy the interstitial positions and the impurity concentrations considered in the present study are small, for impurity added KDP crystals, the impurity molecules can be assumed to be added in the KDP lattice in the same amount (ratio) as estimated. Hence, for impurity added KDP crystals, the total d.c. conductivity increases due to the addition of defects in the form of impurity molecules.

The conductivities are more along the $a$-direction than those along the $c$-direction for both the impurities considered in the present study at low temperatures. This is in correspondence with the results obtained by the previous authors for their systems (Harris and Vella 1966; Shanmugham et al 1985; Freeda and Mahadevan 2000). For the impurity thiourea, for all the impurity concentrations, this is true at all temperatures considered in the present study. However, for the impurity urea the conductivity along $a$-direction becomes less than that along $c$-direction beyond certain temperatures $\left(120^{\circ} \mathrm{C}\right.$ for 0.4 mole $\%, 50^{\circ} \mathrm{C}$ for 0.6 mole $\%$ and $<30^{\circ} \mathrm{C}$ for 0.8 and 1.0 mole $\%$ concentrations).

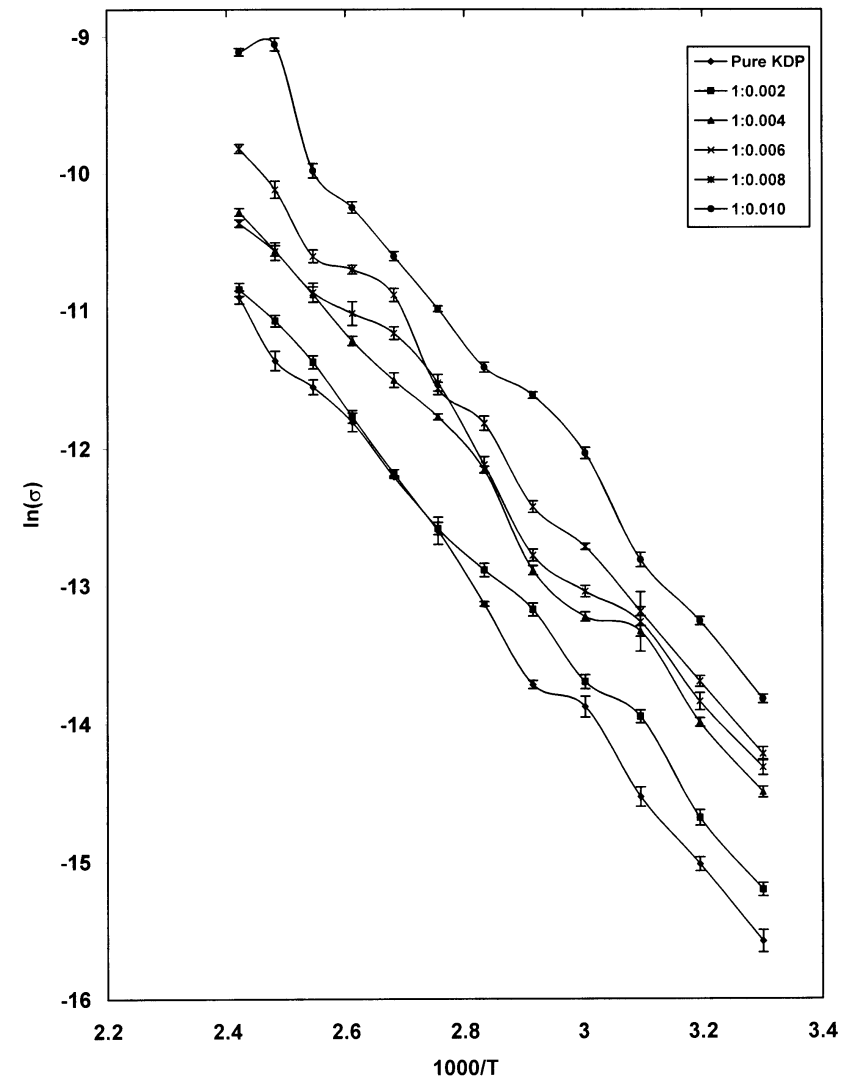

Figure 3. Variation of $\ln (\sigma)$ with $1000 / T$ for thiourea added KDP along $a$-direction. 


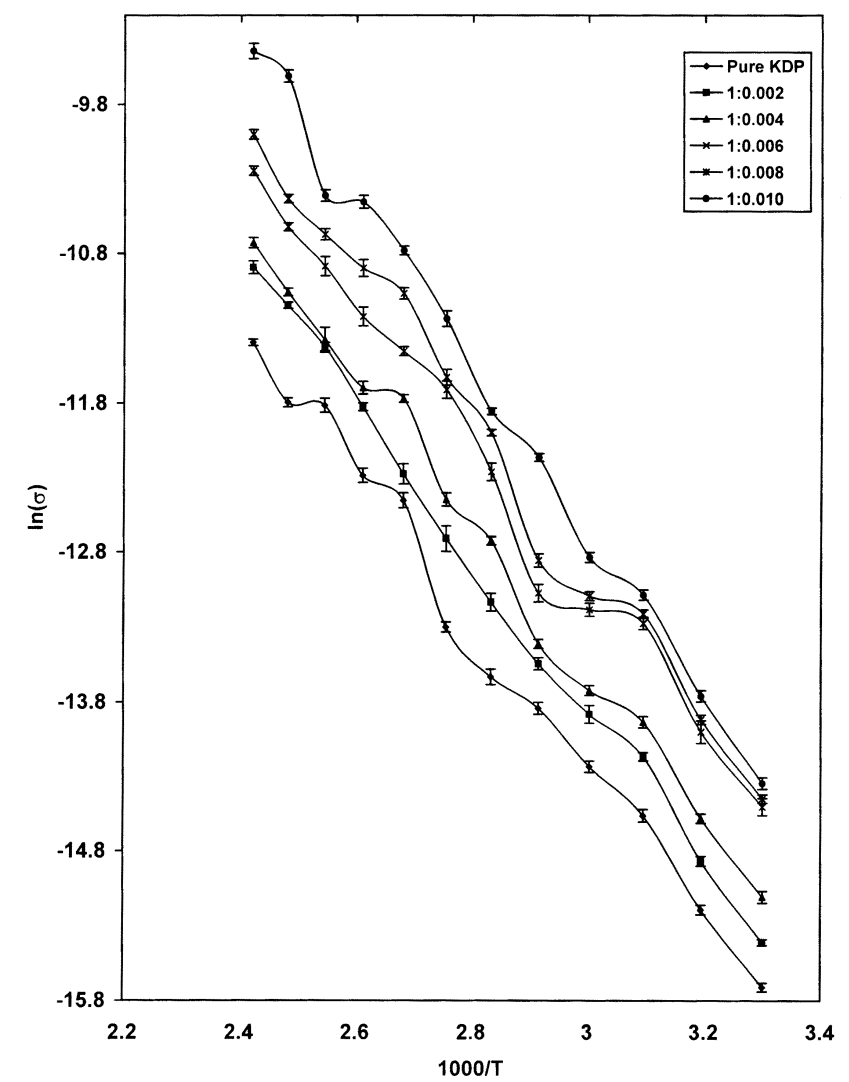

Figure 4. Variation of $\ln (\sigma)$ with $1000 / T$ for thiourea added KDP along $c$-direction.

For both the impurities, the $E$ values are more along $c$ direction than those along $a$-direction for all the impurity concentrations (except for the pure KDP). The activation energy decreases with the increase in impurity concentration in the case of urea while there is no particular order observed in the case of thiourea impurity.

The conduction region considered in the present study seems to be connected to mobility of vacancies. The low activation energies observed suggests that oxygen vacancies may be responsible for conduction in this region. The present study, similar to that by Freeda and Mahadevan (2000), gives further evidence to the statements: (i) the electrical conduction in KDP crystals is protonic; and (ii) the electrical conduction in KDP crystals is mainly due to the anions viz. $\left(\mathrm{H}_{2} \mathrm{PO}_{4}\right)^{-1}$ ions and not the cations viz. $\mathrm{K}^{+}$ions.

\section{Conclusions}

Pure and impurity (urea and thiourea) added KDP single crystals were grown and electrical conductivities were measured along both the $a$ - and $c$-directions at various temperatures ranging from 30 to $140^{\circ} \mathrm{C}$. Density measurement indicates that the impurity molecules have entered into the lattice of KDP crystals. The present study gives further evidence to prove that the conduction in $\mathrm{KDP}$ is protonic and mainly due to the anions and not the cations. The present study indicates that the conductivity increases with the increase in impurity concentration and temperature.

\section{References}

Dean J A (ed.) 1979 Lange's handbook of chemistry (New York: McGraw Hill Book Company) 12 edn

Freeda T H and Mahadevan C 2000 Bull. Mater. Sci. 23335

Harris L B and Vella G J 1966 J. Appl. Chem. 17151

Henisch H K 1988 Crystals in gels and Liesegang rings (Cambridge: Cambridge University Press)

Krishnaswamy R 1984 Crystal growth and characterization of mixed crystals and doped crystals, Proceedings of the second national seminar on crystal growth (Chennai: Anna University) p. 58

Ramasubramanian P S and Mahadevan C 1991 Indian J. Pure \& Appl. Phys. 29285

Rashkovich L N 1991 KDP family single crystals (New York: Adam Hilger)

Shanmugham M, Gnanam F D and Ramasamy P 1985 Indian J. Pure \& Appl. Phys. 2382

Shanthini B, Anitha C, Mahadevan C and Balasingh C 1999 Indian J. Phys. A73 805

Udupa K S, Mohan Rao P, Sriramana Aithal, Bhat A P and Avasthi D K 1997 Bull. Mater. Sci. 201069

Varma K B R, Ramanaiah K V and Rao K V 1983 Bull. Mater. Sci. 539 